\title{
Predicting Pumpability and Shootability of Crushed Aggregate Wet-Mix Shotcrete Based on Rheological Properties
}

\author{
Kyong-Ku Yun, ${ }^{1}$ Pangil Choi, ${ }^{2}$ and Jung Heum Yeon ${ }^{3}$ \\ ${ }^{1}$ Department of Civil Engineering, Kangwon National University, Chuncheon 24341, Republic of Korea \\ ${ }^{2}$ Department of Civil and Environmental Engineering, Texas Tech University, Lubbock, TX 79409, USA \\ ${ }^{3}$ Department of Civil and Environmental Engineering, Gachon University, Seongnam 13120, Republic of Korea
}

Correspondence should be addressed to Jung Heum Yeon; jyeon@gachon.ac.kr

Received 4 October 2016; Accepted 17 October 2016

Academic Editor: Doo-Yeol Yoo

Copyright (C) 2016 Kyong-Ku Yun et al. This is an open access article distributed under the Creative Commons Attribution License, which permits unrestricted use, distribution, and reproduction in any medium, provided the original work is properly cited.

\begin{abstract}
This study aims to estimate the pumpability and shootability of wet-mix shotcrete (WMS) made with crushed aggregates and various admixtures such as silica fume, fly ash, ground granulated blast furnace slag (GGBFS), metakaolin, and steel fiber based on rheological properties. The IBB rheometer was employed as an apparatus to measure the rheological properties of freshly mixed shotcrete such as flow resistance and torque viscosity. Results have shown that the use of silica fume and metakaolin led to satisfactory pumpability, whereas mixtures with fly ash and steel fiber failed to meet the pumping criteria at normal pump pressure. The build-up thickness, an indicator to represent shotcrete shootability, was predicted to vary between 68 and $218 \mathrm{~mm}$, demonstrating that the use of admixtures resulted in a wide spectrum of shootability. In particular, the use of metakaolin was found to substantially increase the predicted build-up thickness only with a small replacement. The findings of this study are expected to be used as an easy-to-use guideline for estimating pumpability and shootability of WMS when no compliance testing data is available.
\end{abstract}

\section{Introduction}

Pumpability and shootability are important functional parameters considered in the design procedure for wet-mix shotcrete (WMS) mixtures [1]. Even if mechanical and durability performance requirements are met, shotcrete may not perform per the design intent without proper pumpability and shootability. Commonly, pumpability of WMS has been quantified via pump pressure and power requirement measurements [2-6]. Because such methods offer visual examinations of the pumpability in real time, relatively accurate and reliable data can be obtained. However, since the measured pump pressure and power requirement are subjected to large variations depending on the pumping equipment used, new compliance testing to assess actual pumpability is necessary whenever there is a change in equipment or process for a given project. This step often involves inefficient, time-consuming, and labor-intensive tasks as the compliance testing needs to be conducted under actual field conditions.
One feasible approach to address this limitation is to indirectly estimate the pumpability through rheological properties of fresh materials, namely, yield stress and plastic viscosity of the Bingham model (or equivalent). Several studies have been conducted to estimate the pumpability of normal- and high-performance shotcrete/concrete based on the rheology and other flow characteristics. Browne and Bamforth [5] attempted to predict the pumpability of fresh concrete based on slump and pressure bleed tests. Beaupre [1] correlated the rheological parameters (i.e., flow resistance and torque viscosity) of WMS to pumpability through piston pressure measurements. Burns [7] characterized WMS pumped through small-diameter hoses by identifying the relationship among rheology, tribology, and pump pressure based on a solid scientific approach. McAskill [8] proposed technical tips to prevent pumping problems during wet-mix process. Seo [9] carried out an experimental study to evaluate the pumpability and fluidity of concrete on the basis of rheological properties. Kwon et al. [10] quantified the pumping characteristics of high-strength concrete by means of 
a continuous pumping system. Yun et al. [11] assessed the rheological properties of WMS with various mineral and chemical admixtures and correlated them to pumpability by measuring piston pump pressure.

Even though many theoretical and practical research attempts to quantify the pumpability of WMS have been made based on prevailing theories of rheology, research studies on shootability predictions have been relatively limited. This is because the shootability is generally subjected to a number of construction uncertainties (such as workmanship of a nozzle operator and field conditions including climates as well as equipment/method used), which made the research approach towards shootability estimation quite challenging [11]. Moreover, most previous studies have been limited to assessing the pumpability and shootability of mixtures with natural aggregate sources such as river gravel. In recognition of the issues, this paper contributes to providing a simple empirical methodology to predict the pumpability and shootability of WMS containing crushed aggregate obtained from tunnel excavation and different type and dosage of admixtures based on rheological measurements, along with performance criteria given in previous research [1]. In addition, dosage ranges for each admixture that provide proper pumpability and shootability were provided to serve as an implementation guideline for WMS. The findings from this study would provide an advanced understanding of fresh WMS behavior, as well as an easy-to-use prediction scheme for pumpability and shootability of WMS.

\section{Experimental}

2.1. Materials. A commercially available Type I portland cement complying with KS L 5201 was employed. The specific gravity and fineness of the portland cement used were 3.15 and $3,289 \mathrm{~cm}^{2} / \mathrm{g}$, respectively. The chemical compositions were as follows: $20.8 \% \mathrm{SiO}_{2}, 6.3 \% \mathrm{Al}_{2} \mathrm{O}_{3}, 61.2 \% \mathrm{CaO}, 3.3 \%$ $\mathrm{MgO}, 2.3 \% \mathrm{SO}_{3}$, and $0.61 \%$ loss-on-ignition (LOI).

Crushed granite obtained from the surrounding rock of tunnel walls was used as coarse aggregate after being sieved and washed. The coarse aggregate used had a maximum aggregate size of $10 \mathrm{~mm}$, a specific gravity of 2.65 , and a fineness modulus of 5.70. As fine aggregate, washed crushed sand with a specific gravity of 2.62 and a fineness modulus of 2.77 was employed. The coarse and fine aggregates used in this study conformed to the gradation specifications of ASTM C33.

A Type I fly ash as per the classification of the Korean Industrial Standards (KS) was used. The chemical properties of the fly ash used were $41.5 \% \mathrm{SiO}_{2}, 16.8 \% \mathrm{C}, 22.9 \% \mathrm{Al}_{2} \mathrm{O}_{3}$, $4.91 \% \mathrm{CaO}$, and $1.84 \% \mathrm{Fe}_{2} \mathrm{O}_{3}$, all of which met the requirements of KS L 5405. The density of the fly ash used was $2.25 \mathrm{~g} / \mathrm{cm}^{3}$, which complied with the specified lower limit of $1.95 \mathrm{~g} / \mathrm{cm}^{3}$.

Silica fume with a density of $3.0-3.5 \mathrm{~g} / \mathrm{cm}^{3}$, a specific surface area of $200,470 \mathrm{~cm}^{2} / \mathrm{g}$, a $0-45 \mu \mathrm{m}$ particle size portion of $98.0 \%$ (by weight), and a moisture content of $1.15 \%$ was employed. The chemical compositions of the silica fume used were as follows: $90.4 \% \mathrm{SiO}_{2}, 2.0 \% \mathrm{CaO}, 3.0 \% \mathrm{Fe}_{2} \mathrm{O}_{3}, 1.5 \%$ $\mathrm{Al}_{2} \mathrm{O}_{3}$, and $2.72 \%$ LOI.
Ground granulated blast furnace slag (GGBFS) with a density of $2.90 \mathrm{~g} / \mathrm{cm}^{3}$, a specific surface area of $4,306 \mathrm{~cm}^{2} / \mathrm{g}$, an activity index at 91 days of 117 , and a flow of $102 \%$ was used. The chemical properties of the GGBFS used were as follows: $5.61 \% \mathrm{MgO}, 1.0 \% \mathrm{SO}_{3}, 0.43 \% \mathrm{LOI}$, and $0.007 \%$ chloride ion.

Metakaolin with a specific gravity of 2.63 and a specific area of $12,000 \mathrm{~cm}^{2} / \mathrm{g}$ was adopted as a mineral admixture. The chemical compositions of the metakaolin used were $56 \%$ $\mathrm{SiO}_{2}, 37 \% \mathrm{Al}_{2} \mathrm{O}_{3}, 2.4 \% \mathrm{Fe}_{2} \mathrm{O}_{3}, 0.2 \% \mathrm{CaO}, 0.3 \% \mathrm{MgO}, 0.8 \%$ $\mathrm{K}_{2} \mathrm{O}$, and $0.2 \% \mathrm{Na}_{2} \mathrm{O}$.

A bundle-type steel fiber with a density of $7.80 \mathrm{~g} / \mathrm{cm}^{3}$, a tensile strength of $1,195.5 \mathrm{MPa}$, and an aspect ratio of $60(\phi 0.5$ $\times 30 \mathrm{~mm}$ ) was used as a reinforcing additive.

2.2. Mixture Proportions. 22 mixtures with a constant waterto-cementitious material ratio $(\mathrm{w} / \mathrm{cm})$ of 0.41 and a fine aggregate-to-total aggregate fraction (S/a) of 0.71 were prepared while varying replacement/addition levels as seen in Table 1. The mixture proportions were chosen based on a survey of previous research [1]. Except for the silica fume mixtures, a $9.1 \%$ silica fume replacement was consistently used to ensure suitable levels of pumpability and shootability, slump $(220 \mathrm{~mm})$, air contents before and after shooting (17 and 5\%, resp.), and 28-day compressive strength $(48 \mathrm{MPa})$. Dosages of sulfonate silica-based airentraining agent and polycarboxylate-based superplasticizer were 0.044 and $4.84 \mathrm{~kg} / \mathrm{m}^{3}$, respectively, which were kept constant among the mixtures.

\subsection{Methods}

2.3.1. Rheometer Test. The IBB rheometer, of which the original and revisited designs were devised at The University of British Columbia and IBB Rheology Inc., respectively, was employed to monitor the rheological properties of freshly mixed shotcrete (see Figure 1). The apparatus is proven to accurately measure the rheological properties of fresh mixtures with inclusions of coarse aggregate $[1,11]$. The testing procedure was as follows:

(1) Calibrate the torque resolution $( \pm 0.5 \mathrm{~N} \cdot \mathrm{m})$ and set the built-in strain gage to a null position.

(2) Pour freshly mixed shotcrete into a 21-liter mixing bowl up to a level of $200 \mathrm{~mm}$.

(3) Lift the mixing bowl until the H-shaped impeller is fully buried in the mixture.

(4) Begin operation with the lowest rotational speed, and gradually increase the speed as required.

During the operation, the torque applied to the rotary impeller as it stirs the mixture with a specified revolving speed was continuously measured by means of a load cell. The testing was conducted under a constant temperature condition of $23^{\circ} \mathrm{C}$ so as to eliminate the unexpected effect of temperature. Figure 2 presents an example of the obtained data, which was fitted by the following form of equation:

$$
T=G+H N
$$


TABLE 1: Mixture proportions of WMS.

\begin{tabular}{|c|c|c|c|c|c|c|c|c|c|}
\hline \multirow{2}{*}{ Admixture } & \multirow{2}{*}{ Replacement $^{1}(\%)$} & \multicolumn{8}{|c|}{ Weight per unit volume $\left(\mathrm{kg} / \mathrm{m}^{3}\right)$} \\
\hline & & SF & FA & GGBFS & MK & $\mathrm{C}$ & $\mathrm{W}$ & $\mathrm{F} / \mathrm{A}$ & $\mathrm{C} / \mathrm{A}$ \\
\hline \multirow{4}{*}{ SF } & 0 & 0 & - & - & - & 440 & 180 & 1159 & 480 \\
\hline & 5 & 22 & - & - & - & 418 & 180 & 1153 & 478 \\
\hline & 9.1 & 40 & - & - & - & 400 & 180 & 1149 & 476 \\
\hline & 15 & 66 & - & - & - & 374 & 180 & 1142 & 473 \\
\hline \multirow{4}{*}{ FA } & 0 & 40 & 0 & - & - & 400 & 180 & 1149 & 476 \\
\hline & 10 & 40 & 44 & - & - & 356 & 180 & 1137 & 471 \\
\hline & 20 & 40 & 88 & - & - & 312 & 180 & 1125 & 466 \\
\hline & 30 & 40 & 132 & - & - & 268 & 180 & 1113 & 461 \\
\hline \multirow{5}{*}{ GGBFS } & 0 & 40 & - & 0 & - & 400 & 180 & 1149 & 476 \\
\hline & 10 & 40 & - & 44 & - & 356 & 180 & 1146 & 475 \\
\hline & 20 & 40 & - & 88 & - & 312 & 180 & 1144 & 474 \\
\hline & 30 & 40 & - & 132 & - & 268 & 180 & 1142 & 473 \\
\hline & 40 & 40 & - & 176 & - & 224 & 180 & 1140 & 472 \\
\hline \multirow{4}{*}{ MK } & 0 & 40 & - & - & 0 & 400 & 180 & 1149 & 476 \\
\hline & 5 & 40 & - & - & 22 & 378 & 180 & 1146 & 475 \\
\hline & 10 & 40 & - & - & 44 & 356 & 180 & 1144 & 474 \\
\hline & 15 & 40 & - & - & 66 & 334 & 180 & 1141 & 473 \\
\hline \multirow{5}{*}{ Steel fiber } & $0^{2}$ & 40 & - & - & - & 400 & 180 & 1149 & 476 \\
\hline & $30^{2}$ & 40 & - & - & - & 400 & 180 & 1149 & 476 \\
\hline & $40^{2}$ & 40 & - & - & - & 400 & 180 & 1149 & 476 \\
\hline & $50^{2}$ & 40 & - & - & - & 400 & 180 & 1149 & 476 \\
\hline & $60^{2}$ & 40 & - & - & - & 400 & 180 & 1149 & 476 \\
\hline
\end{tabular}

Notes. $\mathrm{SF}$ = silica fume; FA = fly ash; GGBFS = ground granulated blast furnace slag; $\mathrm{MK}=$ metakaolin; $\mathrm{C}=$ cement; $\mathrm{W}=$ water; $\mathrm{F} / \mathrm{A}=$ fine aggregate; and $\mathrm{C} / \mathrm{A}$ $=$ coarse aggregate.

${ }^{1} \%$ by weight of total cementitious material content.

${ }^{2}$ A given weight per unit volume $\left(\mathrm{kg} / \mathrm{m}^{3}\right)$ of steel fibers was added to mixtures.

in which $T$ is the torque exerted on the rotary impeller $(\mathrm{N} \cdot \mathrm{m})$; $G$ is the flow resistance $(\mathrm{N} \cdot \mathrm{m}) ; H$ is the torque viscosity $(\mathrm{N} \cdot \mathrm{m} \cdot \mathrm{s})$; and $\mathrm{N}$ is the angular speed of the rotary impeller $(\mathrm{rev} / \mathrm{s})$; in other words, the slope of the result plot denotes the torque viscosity, while the $y$-intercept denotes the flow resistance.

The torque viscosity and flow resistance have comparable physical meanings to yield stress and plastic viscosity, respectively, by definition of the Bingham model as follows [12]:

$$
\begin{aligned}
\tau_{0} & =136 G, \\
\mu & =15.2 H,
\end{aligned}
$$

where $\tau_{0}$ is the yield stress $(\mathrm{Pa}) ; G$ is the flow resistance $(\mathrm{N} \cdot \mathrm{m})$; $\mu$ is the plastic viscosity (Pa.s); and $H$ is the torque viscosity $(\mathrm{N} \cdot \mathrm{m} \cdot \mathrm{s})$.

2.3.2. Pumpability Predictions. The pumpability of WMS was estimated based on previously published data by Beaupre [1]. Beaupre correlated the pumpability of various shotcrete mixtures (total 42 mixtures) with and without steel fibers subjected to different levels of pump pressure to their rheological parameters and then determined borderline values for flow resistance and torque viscosity, as shown in Figure 3.
If a given mixture had flow resistance and torque viscosity smaller than the suggested borderline values (flow resistance $G=4.3 \mathrm{~N} \cdot \mathrm{m}$ and torque viscosity $H=2.9 \mathrm{~N} \cdot \mathrm{m} \cdot \mathrm{s}$ ) at the same time, the mixture was considered "pumpable," whereas it was considered "nonpumpable" if either or both were greater than the borderline values.

2.3.3. Shootability Predictions. The shootability of WMS is often characterized by a build-up thickness. In this study, flow resistance was chosen as an indicator to predict the build-up thickness of WMS because the build-up thickness had a fairly proportional relationship to flow resistance (see Figure 4(a)), whereas it exhibited a quite random relationship with torque viscosity, particularly within the torque viscosity range of 0 $1 \mathrm{~N} \cdot \mathrm{m} \cdot \mathrm{s}$, as shown in Figure $4(\mathrm{~b})$.

Two different relationships presented in former studiesone based on the two-point test [13] and the other based on the zero-speed measurement [1] - were used to construct a single equation that describes the relationship between the build-up thickness and flow resistance. As noted in Figure 4(a), the regression equation encompassing the two data sets yielded a high level of coefficient of determination of over 0.85 , which would be reliably used to predict the build-up thickness once the flow resistance is determined via laboratory experiments. 


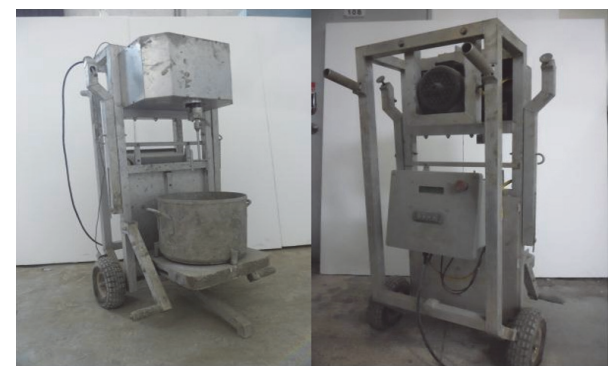

(a)

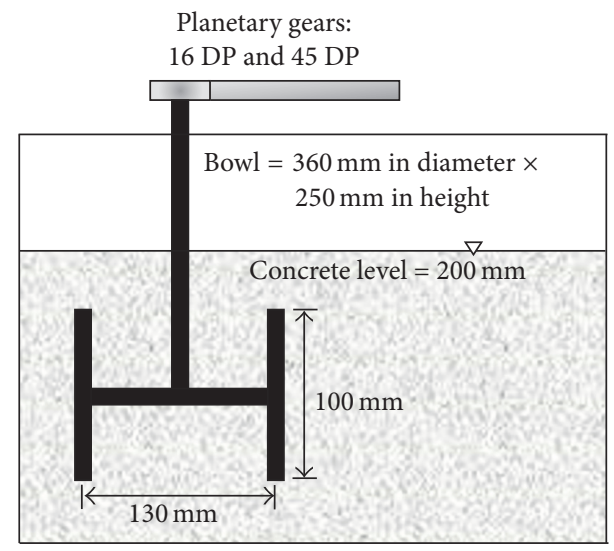

(b)

FIGURE 1: IBB rheometer: (a) front and rear view and (b) schematic diagram.

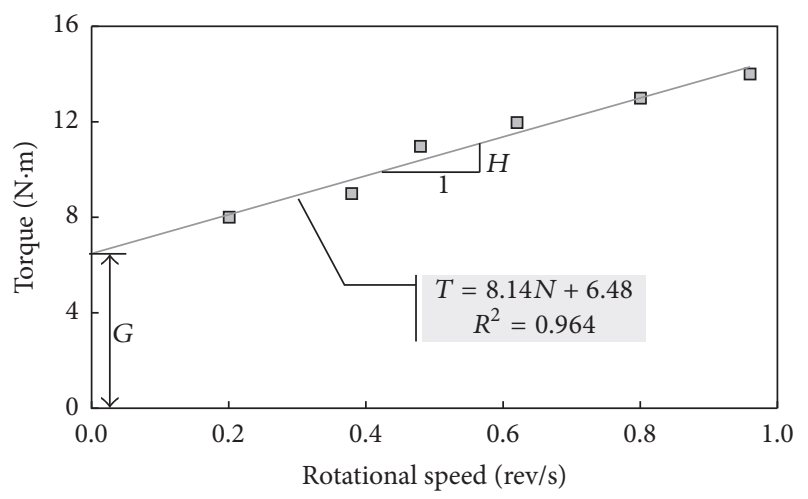

FIgURE 2: Typical data obtained from the IBB rheometer.

\section{Results and Discussion}

\subsection{Rheological Behavior}

3.1.1. Results. The results of rheometer tests for various silica fume mixtures are shown in Figure 5. The results indicate that increasing the silica fume replacement from 0 to $15 \%$ gradually increased the $y$-intercept of the regression line, thereby increasing the flow resistance. On the other hand, the silica fume replacement led to a general reduction in torque viscosity, but this reduction effect was pronounced only when

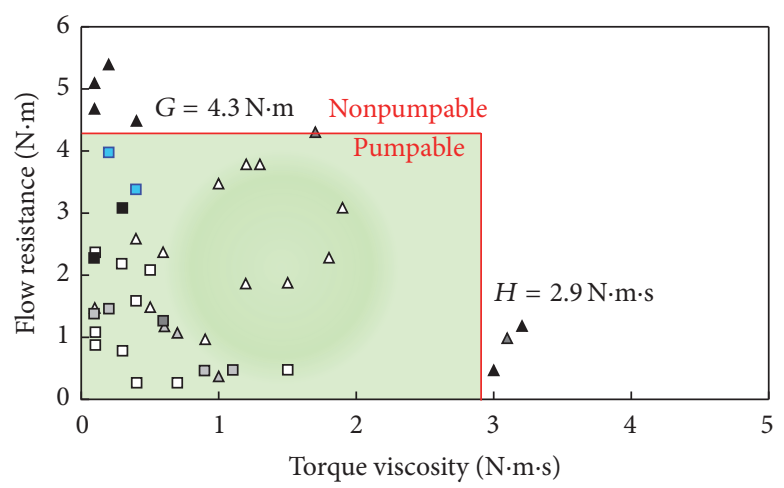

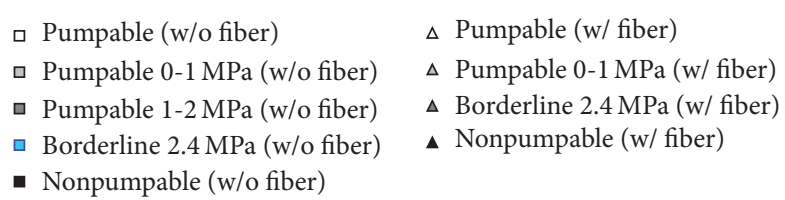

FIGURE 3: Rheological properties for different mixtures and their borderline values [1].

a $5 \%$ silica fume replacement was used, which was different from the findings of previous studies $[1,14]$.

Figure 6 shows the applied torque and angular speed relationships for mixtures containing fly ash with a $9.1 \%$ silica fume replacement. Note that the slope of the regression line significantly increased upon fly ash replacements, thereby increasing the torque viscosity. The rate of slope increase tended to decrease with increased fly ash replacement. Also, the use of fly ash led to a general increase in flow resistance by raising the $y$-intercept of the regression line. No evident difference in flow resistance was found between 20 and $30 \%$ mixtures.

The experimental data and their regression lines for GGBFS mixtures are presented in Figure 7. It is evident from the results that the GGBFS replacements lowered the flow resistance by shifting the regression line downwards along the $y$-axis, which may reduce the build-up thickness. In particular, a substantial reduction in flow resistance was detected when a $10 \%$ GGBFS replacement was used, even though it was quite difficult to discern evident discrepancies across the 20,30, and $40 \%$ mixtures. There were no clear differences in the slope of the regression line among the GGBFS mixtures.

Figure 8 shows how the applied torque was varied to obtain prescribed impeller angular speeds for metakaolin mixtures with $9.1 \%$ silica fume. Note that no noticeable changes in flow resistance and torque viscosity were observed when 5\% metakaolin was used compared with nonmetakaolin mixture. However, the flow resistance dramatically increased upon more than $10 \%$ metakaolin replacements, thereby increasing the predicted build-up thickness. A significant increase in torque viscosity was obtained when $15 \%$ metakaolin was used. This behavior is most likely attributed to the large specific surface area of the metakaolin used and eventual microfiller effect (i.e., dense packing of the cement matrix) [15]. 


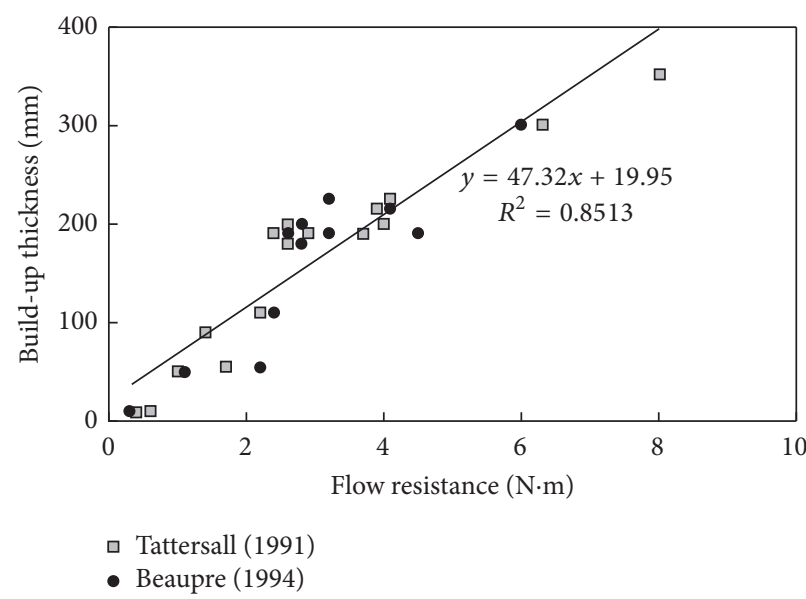

(a)

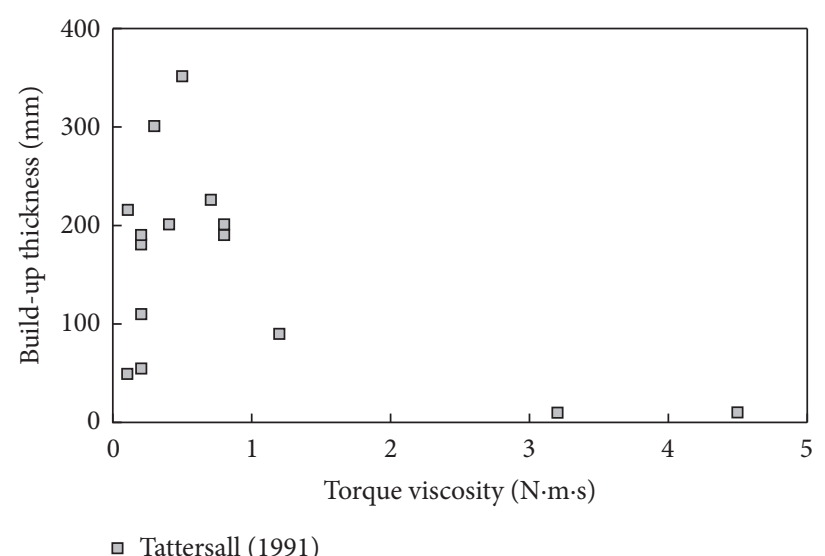

(b)

FIGURE 4: Relationships between rheological parameters and build-up thickness: (a) flow resistance versus build-up thickness; (b) torque viscosity versus build-up thickness [1].

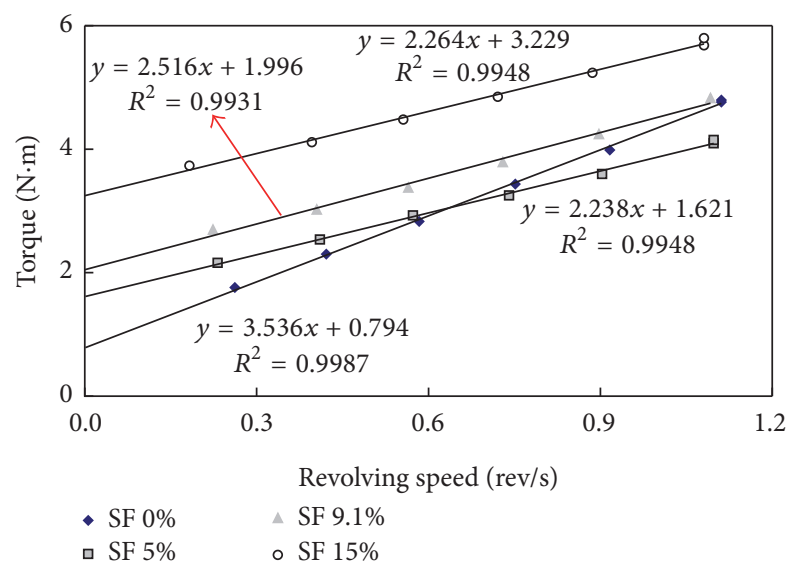

FIGURE 5: Exerted torque versus rotational speed relationship for silica fume mixtures.

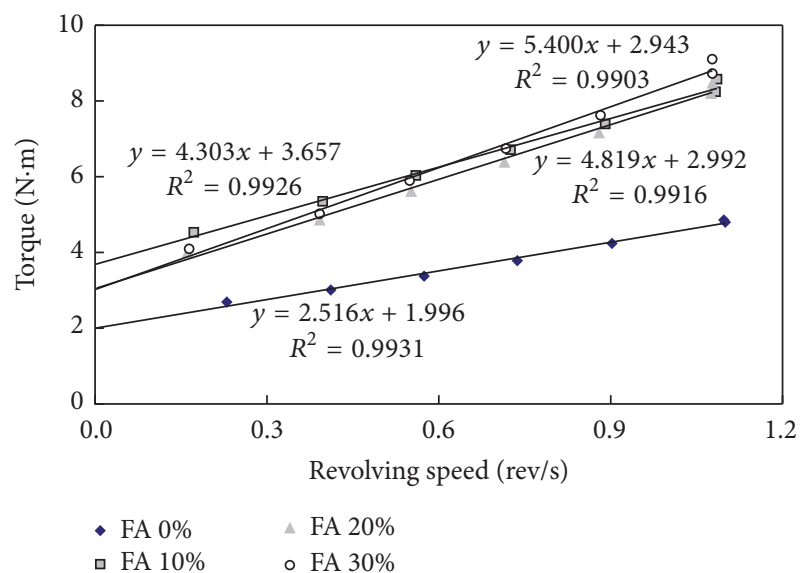

FIGURE 6: Exerted torque versus rotational speed relationship for fly ash mixtures with $9.1 \%$ silica fume.

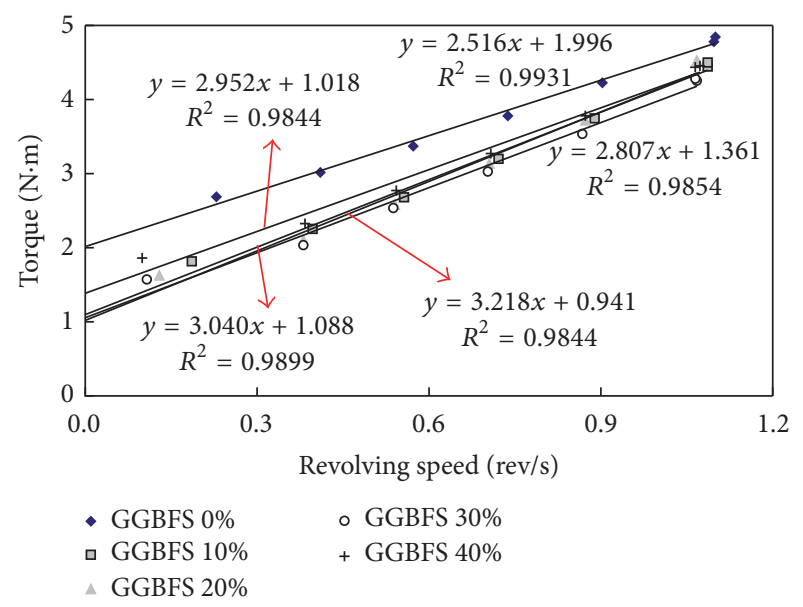

FIGURE 7: Exerted torque versus rotational speed relationship for GGBFS mixtures with $9.1 \%$ silica fume.

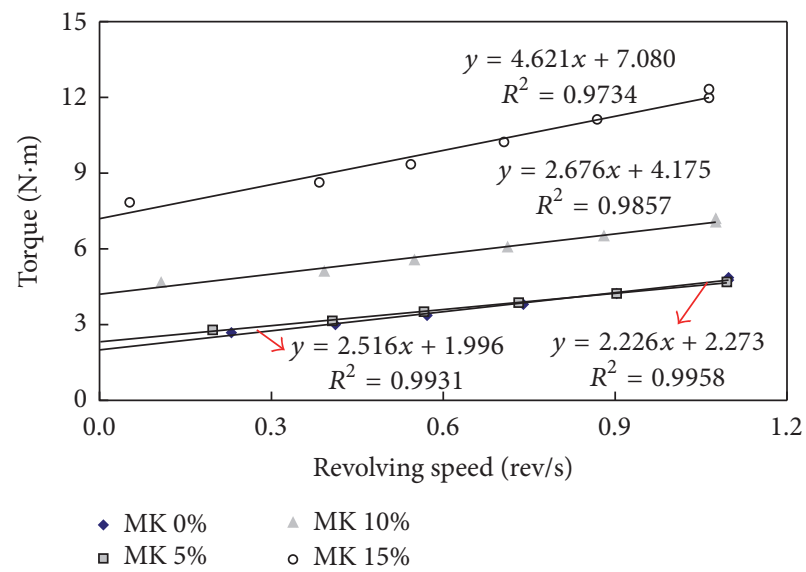

FIGURE 8: Exerted torque versus rotational speed relationship for metakaolin mixtures with $9.1 \%$ silica fume. 


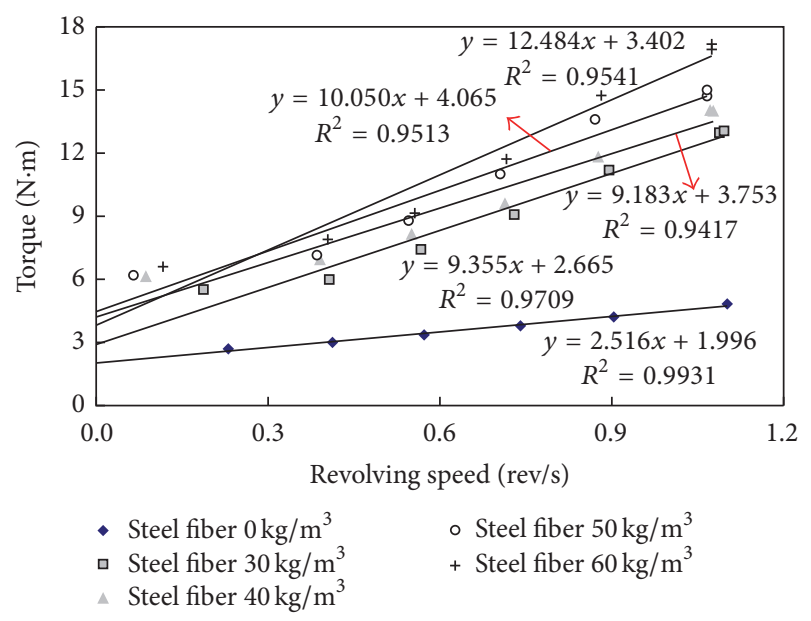

FIgURE 9: Exerted torque versus rotational speed relationship steel fiber mixtures with $9.1 \%$ silica.

The rheometer test results for steel fiber mixtures with a 9.1\% silica fume replacement are given in Figure 9. As the results show, the regression lines for steel fiber mixtures were all above the regression line for non-steel fiber reinforced mixture, indicating that the steel fiber additions increased the flow resistance of WMS even though the trend was not consistent. Also noted is that the use of steel fibers resulted in regression lines with a greater slope, thereby reducing the pumpability of WMS. The pumpability reduction effect (or the torque viscosity-increasing effect) tended to become more pronounced when a high dosage $\left(>50 \mathrm{~kg} / \mathrm{m}^{3}\right)$ of steel fibers was used, which is quite unfavorable for WMS applications.

3.1.2. Discussion. Figures 10 (a) and 10(b) illustrate the variations in flow resistance and torque viscosity depending on the admixture type and replacement level, drawn based on Figures 5-9. It is remarkable that the use of silica fume led to an overall decrease in torque viscosity, while increasing the flow resistance almost in proportion to the replacement level. This behavior would play a beneficial role in both pumpability and shootability, especially when applied to a project where strict build-up thickness control is required; for example, a silica fume replacement should be minimized when multiplelayer spraying is allowed, while it should be increased as required when single-layer spraying is needed. The fly ash replacement was found to worsen the pumpability of WMS by markedly increasing the torque viscosity, while improving the shootability. The use of GGBFS had a negligible effect on the pumpability by maintaining a similar level of torque viscosity, while reducing the shootability. Metakaolin was predicted to have the most significant effect on the shootability increase particularly when more than $10 \%$ replacement was used, although a substantial increase in torque viscosity occurred upon $15 \%$ replacement. Accordingly, a maximum of $10 \%$ replacement is recommended to exploit the benefits of metakaolin in shootability, without adversely affecting the pumpability. Steel fiber additions were likely to cause severe pumping problems as the torque viscosity of steel fiber mixtures was 3.6 to 5 times as high as that of controlled mixture. Thus, caution should be taken when steel fibers are selected as a reinforcing additive for WMS.

\subsection{Pumpability Predictions Based on Rheological Properties.} Figure 11 graphically summarizes the results of pumpability prediction for each WMS mixture, in which the area surrounded by the red dotted lines is the "pumpable" zone identified by the previous study [1]. The zoom-in view around the "pumpable" zone is also given in the top right corner of the plot. The results have shown that three silica fume mixtures (i.e., 5, 9.1, and 15\% mixtures) fell within the "pumpable" zone, meeting the suggested threshold values for rheological parameters (which enables WMS to be pumped at normal pump pressure) while non-silica fume mixture failed to meet the criteria. This was obtained most probably because an adequate amount of spherical silica fume particles incorporated promoted the ball-bearing effect and prevented the drainage of lubricating water from the cement paste matrix, lowering the torque viscosity of the mixtures [16]. Also noted is that the flow resistance increment between 5 and $15 \%$ mixtures was about twice that between 5 and $9.1 \%$ mixtures, while maintaining a similar level of torque viscosity. This implies that, as previously mentioned, the use of silica fume is quite effective in increasing the build-up thickness, even without the use of accelerator.

Furthermore, the results of pumpability prediction for fly ash mixtures are presented in Figure 11. It can be seen that the pumpable condition was achieved only when no fly ash was used, while all mixtures with fly replacements (i.e., 10, 20 , and $30 \%$ ) fell on the "nonpumpable" zone. To make the fly ash mixtures pumpable by reducing the torque viscosity, an air-entraining agent (AEA) may be used [17], but the use of AEA was found to rather decrease the pumpability and air content because part of the AEA was adsorbed by unburned carbon in fly ash. To obtain the required pumpability, thus, a greater dosage of AEA is recommended in consideration of the AEA losses due to adsorption, as well as the fineness of the fly ash used.

When GGBFS was used, two mixtures (i.e., 0 and 40\%) successfully obtained the pumpable condition, while other mixtures failed to meet the pumping requirements. However, it appears that the GGBFS itself had a nonsignificant effect on the pumpability because the variations in torque viscosity and flow resistance per the replacement level were quite small compared with other mixture groups. This, in turn, implies that the high fineness of the GGBFS used $\left(4,310 \mathrm{~cm}^{2} / \mathrm{g}\right)$ had a minimal effect on the rheological properties of WMS.

The results given in Figure 11 also show how the use of metakaolin affected the pumpability of WMS. It should be noted that the $5 \%$ metakaolin mixture rather had smaller torque viscosity and greater flow resistance compared to nonmetakaolin mixture. The metakaolin mixtures were found to satisfy the pumpable criteria until the replacement level increased up to $10 \%$, which demonstrates that metakaolin can be a good alternative or complement to silica fume. However, because more than $10 \%$ metakaolin replacement may degrade the pumpability by providing a substantial 


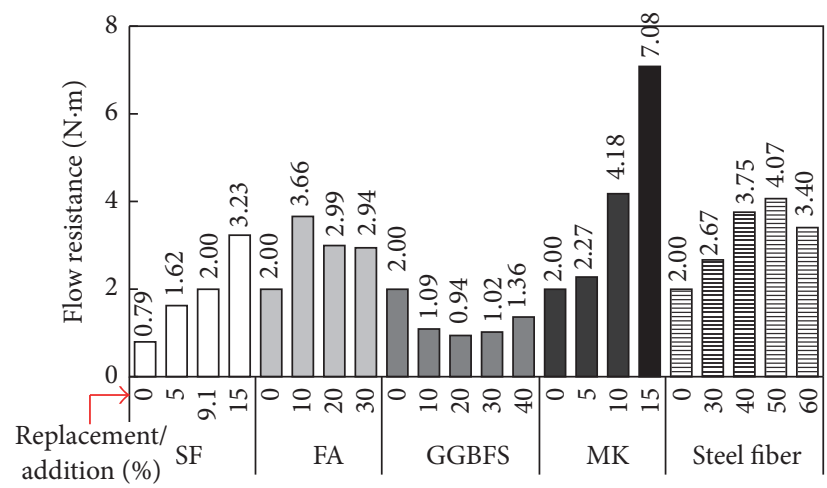

(a)

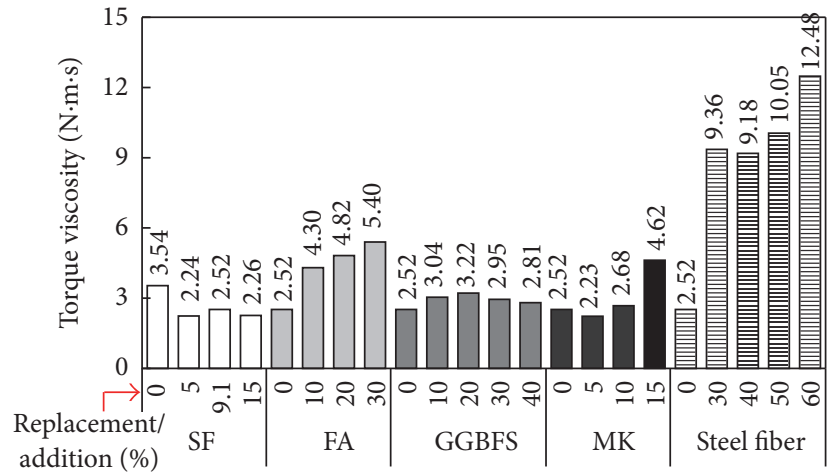

(b)

FIGURE 10: Variations in rheological properties for various WMS mixtures: (a) variations in flow resistance and (b) variations in torque viscosity.

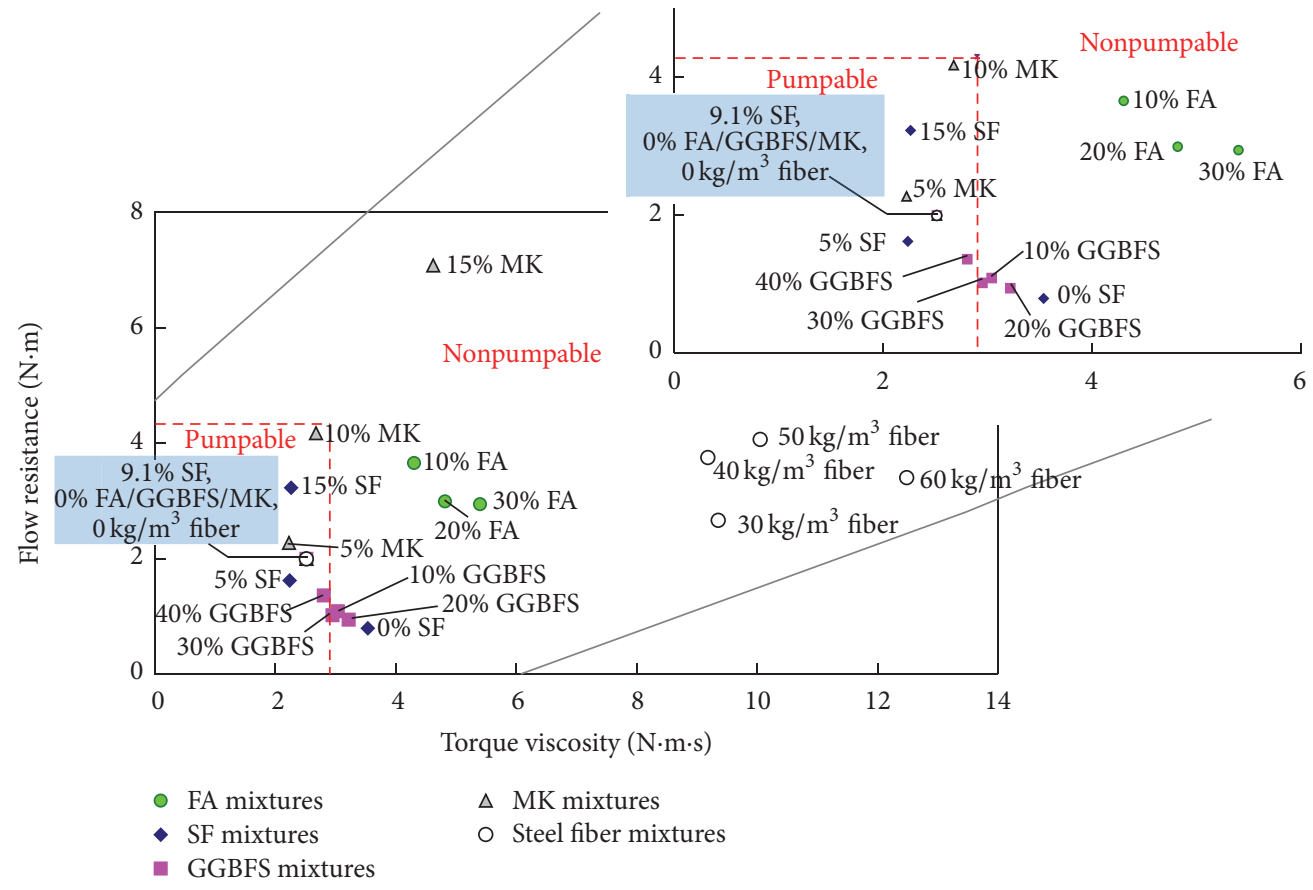

FIGURE 11: Pumpability predictions for various WMS mixtures.

increase in both torque viscosity and flow resistance, no more than $10 \%$ replacement is recommended when metakaolin is chosen as a mineral admixture for WMS.

All mixtures with steel fibers were found to fall on the "nonpumpable" zone. This is because the steel fiber additions enormously increased the torque viscosity as high as 9.18$12.48 \mathrm{~N} \cdot \mathrm{m} \cdot \mathrm{s}$-about 3.17-4.3 times greater than the border line value for torque viscosity-while keeping a similar level of initial slump (i.e., 215-230 mm). Accordingly, when steel fibers are to be used for a shotcrete project, compliance testing may need to be conducted prior to field practice to check the actual pumpability.

Table 2 provides the recommended replacement/addition ranges for each mixture group determined based on the
TABLE 2: Recommended replacement/addition ranges for each admixture.

\begin{tabular}{lcc}
\hline Mixture group & Pumpable (\%) & Nonpumpable (\%) \\
\hline Silica fume only & $5-15$ & $<5$ \\
Fly ash & - & All \\
GGBFS & $30-40$ & $<30$ \\
9.1\% silica fume & & \\
Metakaolin & $0-10$ & $>10$ \\
Steel fiber & - & All \\
\hline
\end{tabular}

rheometer test results and data from the previous study [1]. While some of silica fume, GGBFS, and metakaolin mixtures 


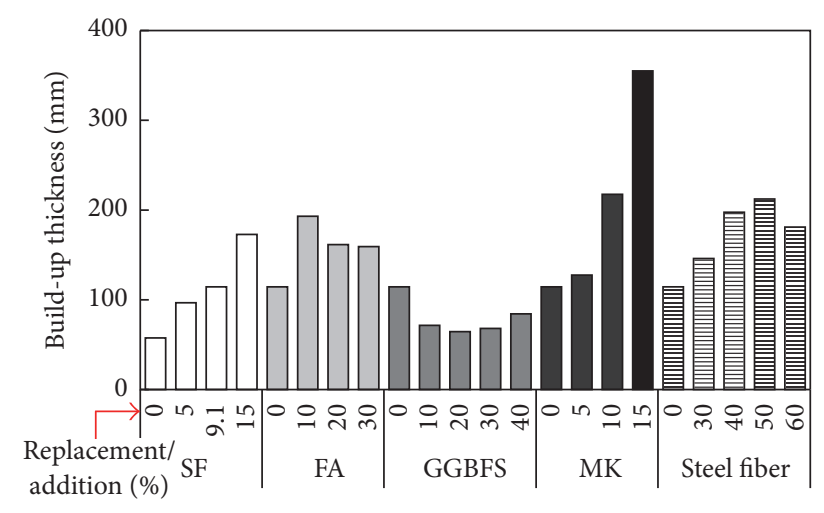

FIGURE 12: Shootability (build-up thickness) predictions for various WMS mixtures.

met the pumpable criteria, fly ash and steel fiber mixtures were all unpumpable for entire replacement/addition ranges. In particular, silica fume and metakaolin mixtures tended to meet the requirements only with relatively small replacement levels, which implies that silica fume and metakaolin may be used in WMS with good expected pumping performance. To implement those materials in actual shotcrete projects, additional research is needed to better understand how silica fume and metakaolin affect other material properties such as mechanical properties and durability.

3.3. Shootability Predictions Based on Flow Resistance. The build-up thickness of various WMS mixtures, as a measure of shootability, was predicted using the regression equation derived in Figure 4(a) and measured flow resistance, and the results are presented in Figure 12. The predicted buildup thickness for silica fume mixtures tended to increase as the silica fume replacement increased because the use of silica fume led to an increase in flow resistance. The shootability of $9.1 \%$ mixture was found to be worse than expected, because there was only a small increment in buildup thickness between 5 and 9.1\% mixtures, compared with that between 0 and $5 \%$. Also found is that the rate of build-up thickness increase substantially increased when the silica fume replacement exceeded $10 \%$, which implies that modifications to mixture proportions may be needed for the control of build-up thickness when more than $10 \%$ silica fume replacement is expected.

The maximum build-up thickness for fly ash mixtures was obtained when $10 \%$ fly ash was used. The build-up thickness then decreased as the fly ash replacement increased above $10 \%$. The inconsistent trend of build-up thickness changes according to the replacement level may be due to the effect of different air contents among the mixtures-the air contents for $0,10,20$, and $30 \%$ fly ash mixtures were $18,15,11$, and $10 \%$, respectively. A further investigation needs to be conducted to clearly identify the sole influence of flow resistance on the build-up thickness.

The build-up thickness of GGBFS mixtures was predicted as $65-114 \mathrm{~mm}$, which was relatively smaller than that of other mixtures. This is most likely because, as previously described,
TABLE 3: Predicted build-up thickness ranges for WMS mixtures meeting pumping requirements.

\begin{tabular}{lcc}
\hline Mixture group & Pumpable range (\%) & $\begin{array}{c}\text { Predicted build-up } \\
\text { thickness }(\mathrm{mm})\end{array}$ \\
\hline $\begin{array}{l}\text { Silica fume only } \\
\text { Fly ash }\end{array}$ & $5-15$ & $97-173$ \\
GGBFS & - & - \\
9.1\% silica fume & $30-40$ & $68-84$ \\
Metakaolin & $0-10$ & $114-218$ \\
Steel fiber & - & - \\
Overall range & $5-15$ & $68-218$ \\
\hline
\end{tabular}

GGBFS gave rise to the ball-bearing effect, thereby increasing the workability of mixtures. Given the relatively smaller build-up thicknesses and little effect of GGBFS replacement level on the build-up thickness, it appears that GGBFS is not a suitable admixture for shootability improvements.

Metakaolin mixtures led to an overall increase in predicted build-up thickness with an increased metakaolin replacement. Specifically, a substantial increase in build-up thickness was observed when the replacement level became greater than $5 \%$. This implies that caution should be taken to control the build-up thickness when proportioning shotcrete mixtures with more than $5 \%$ metakaolin, while up to $5 \%$ metakaolin can be used without essential modifications to mixture proportions.

The predicted build-up thickness for steel fiber mixtures was found to increase until the addition level reached up to $50 \mathrm{~kg} / \mathrm{m}^{3}$, whereas a noticeable reduction was observed at a higher addition level beyond $50 \mathrm{~kg} / \mathrm{m}^{3}$. Even though the build-up thicknesses predicted were overall sufficient for the use in actual practice, however, steel fiber may not be a suitable additive given the poor pumpability of WMS, as previously discussed.

Table 3 summarizes the ranges of predicted build-up thickness for each mixture group meeting the pumpability requirements. The results show that the predicted buildup thickness of $97-173 \mathrm{~mm}$ was achieved with silica fume replacements only. Metakaolin with 9.1\% silica fume enabled the build-up thickness to vary in a broad range between 114 and $218 \mathrm{~mm}$, whereas GGBFS with $9.1 \%$ silica fume resulted in only small variations in build-up thickness between 68 and $84 \mathrm{~mm}$. In particular, a $10 \%$ metakaolin replacement provided a build-up thickness of up to $218 \mathrm{~mm}$, which could be suitably used for massive construction. Build-up thickness predictions for fly ash and steel fibers mixtures could not be made since they did not comply with the pumping criteria at all. Based on the findings, metakaolin, when combined with silica fume, appears to be a promising admixture that effectively enhances both pumpability and shootability even with a small replacement level (i.e., less than 10\%).

\section{Conclusions and Recommendations}

In this paper, the pumpability and shootability of wetmix shotcrete (WMS) with crushed aggregates and various 
admixtures were estimated based on a series of rheometer tests. Optimum replacement/addition ranges for each admixture were also proposed as a guideline for actual practice, along with expected build-up thicknesses upon shooting. Based on the findings of this study, the following conclusions can be made:

(i) Silica fume had positive effects on both pumpability and shootability by decreasing torque viscosity and increasing flow resistance simultaneously.

(ii) Fly ash replacements increased the torque viscosity of WMS, which may lead to poor pumpability.

(iii) Ground granulated blasted furnace slag (GGBFS) replacements tended to reduce the shootability of WMS, while it had a nonsignificant effect on the pumpability.

(iv) Metakaolin replacements significantly increased the shootability of WMS, while keeping a similar level of pumpability across the mixtures.

(v) Adding steel fibers resulted in a substantial increase in torque viscosity, which may not be a good strategy to enhance the pumping performance.

(vi) While all fly ash and steel fiber mixtures failed to meet the pumping requirements, several silica fume, GGBFS, and metakaolin mixtures complied with the pumping criteria at normal pump pressure. In particular, silica fume and metakaolin mixtures were found to have good pumping performance.

(vii) A wide range of build-up thicknesses between 68 and $218 \mathrm{~mm}$ was obtained for WMS mixtures with various type and dosage of admixtures. Particularly, the use of metakaolin dramatically increased the build-up thickness only with a $10 \%$ replacement.

The results outlined in this study would be used as a simple estimate of the pumpability and shootability predictions, as far as no compliance testing is conducted for a given project. A little more conservative approach is recommended taking into account the errors that may be caused by measurements and material variability. Future research will be performed to understand how the use of those admixtures plays a role in actual pumpability and shootability.

\section{Competing Interests}

The authors declare that there is no conflict of interests regarding the publication of this paper.

\section{Acknowledgments}

This research was supported by both grant A (13RDRPB066780) and grant B (15TLRP-B079261-02), funded by Ministry of Land, Infrastructure and Transport of Korean government, and was partly supported by Kangwon National University (Grant no. 120131837), Chuncheon, Republic of Korea.

\section{References}

[1] D. Beaupre, Rheology of high performance shotcrete [Ph.D. thesis], University of British Columbia, Vancouver, Canada, 1994.

[2] O. Dawson, "Pumping concrete-friction between concrete and pipeline," Magazine of Concrete Research, vol. 1, pp. 135-140, 1945.

[3] A. N. Ede, "The resistance of concrete pumped through pipelines," Magazine of Concrete Research, vol. 9, no. 27, pp. 129140,1957

[4] J. E. Gary, "Laboratory procedure for comparing pumpability of concrete mixtures," Proceedings of ASTM, vol. 62, pp. 964-971, 1962.

[5] R. D. Browne and P. B. Bamforth, "Test to establish concrete pumpability," ACI Journal, vol. 74, pp. 193-203, 1977.

[6] G. M. Idorn, "Rheology in fresh concrete," in Proceedings of the Materials Research Society Symposium, Boston, Mass, USA, November 1982.

[7] D. Burns, Characterization of wet-mix shotcrete for small line pumping [M.S. thesis], University of Laval, Quebec City, Canada, 2008.

[8] N. McAskill, "Overcoming pumping problems," American Shotcrete Association Magazine, vol. 2, no. 4, pp. 22-23, 2000.

[9] I. Seo, Study on the evaluation of pumpability and fluidity of concrete using rheological parameters [M.S. thesis], Hanyang University, Ansan, South Korea, 2010.

[10] D.-H. Kwon, H.-S. Lee, J.-Y. Jeon, W.-T. Jeong, H.-K. Jo, and H.R. Kim, "Evaluation of pumping characteristics of high strength concrete using continuous pumping system," Journal of the Korea Institute of Building Construction, vol. 11, no. 4, pp. 387395, 2011.

[11] K.-K. Yun, P. Choi, and J. H. Yeon, "Correlating rheological properties to the pumpability and shootability of wet-mix shotcrete mixtures," Construction and Building Materials, vol. 98, pp. 884-891, 2015.

[12] G. H. Tattersall and P. F. G. Banfill, The Rheology of Fresh Concrete, Chapman \& Hall, London, UK, 1983.

[13] G. H. Tattersall, Workability and Quality Control of Concrete, Chapman \& Hall, London, UK, 1991.

[14] J. Wolsiefer and D. R. Morgan, "Silica fume in shotcrete," Concrete International, vol. 15, no. 4, pp. 34-39, 1993.

[15] P. Dinakar, P. K. Sahoo, and G. Sriram, "Effect of metakaolin content on the properties of high strength concrete," International Journal of Concrete Structures and Materials, vol. 7, no. 3, pp. 215-223, 2013.

[16] E. Sakai, Y. Kakinuma, K. Yamamoto, and M. Daimon, "Relation between the shape of silica fume and the fluidity of cement paste at low water to powder ratio," Journal of Advanced Concrete Technology, vol. 7, no. 1, pp. 13-20, 2009.

[17] K.-K. Yun, S.-Y. Choi, and J. H. Yeon, "Effects of admixtures on the rheological properties of high-performance wet-mix shotcrete mixtures," Construction and Building Materials, vol. 78, pp. 194-202, 2015. 

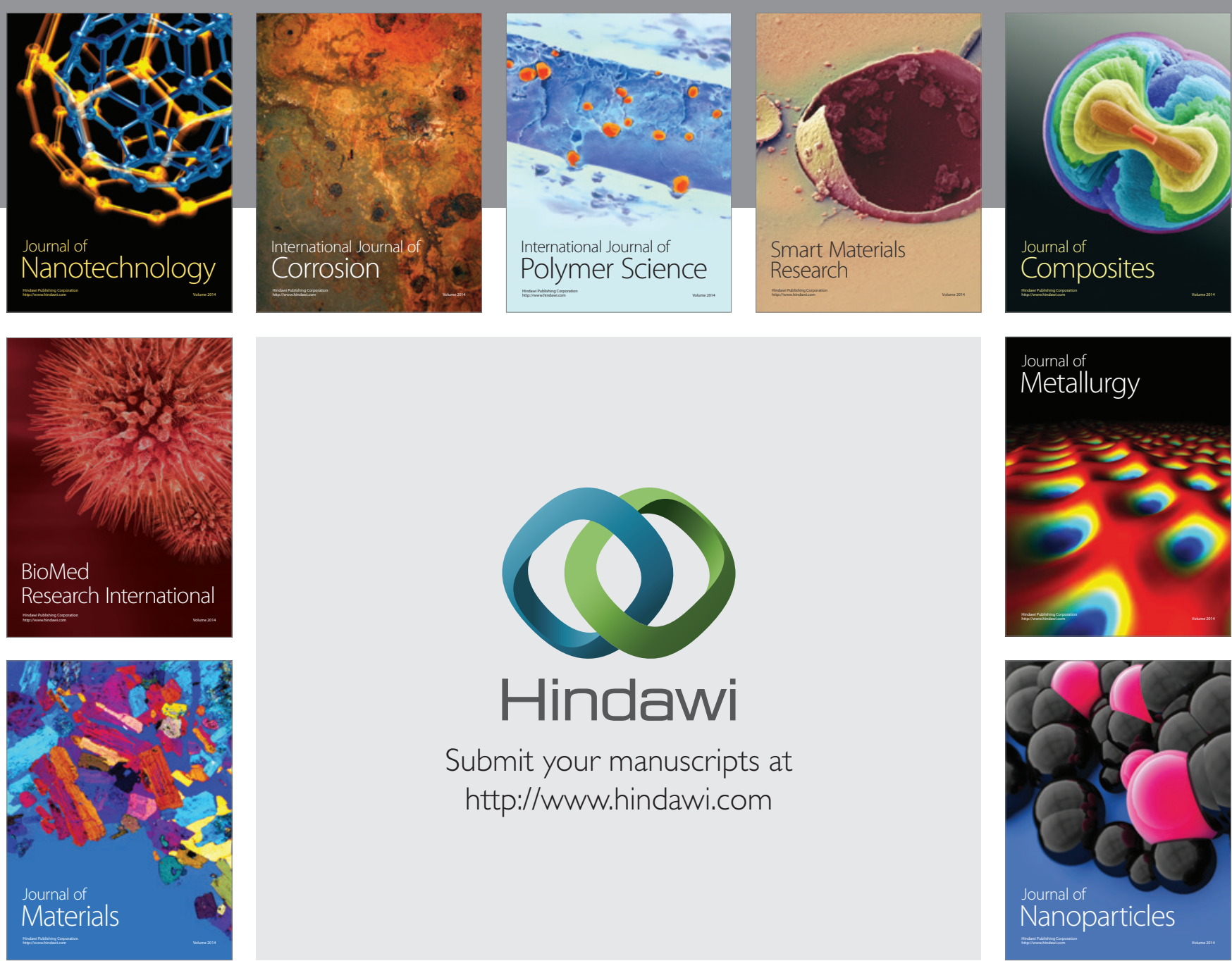

\section{Hindawi}

Submit your manuscripts at

http://www.hindawi.com

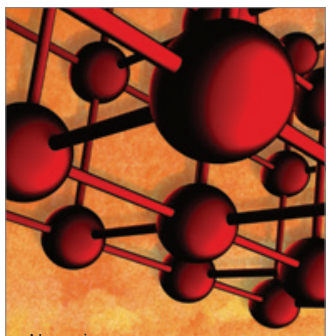

Materials Science and Engineering
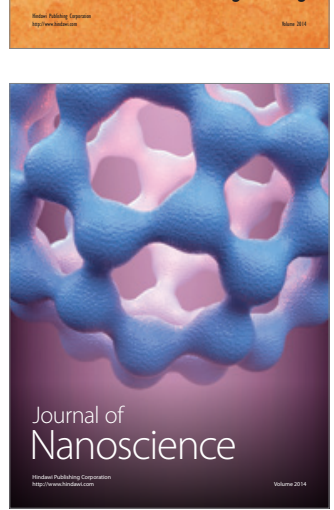
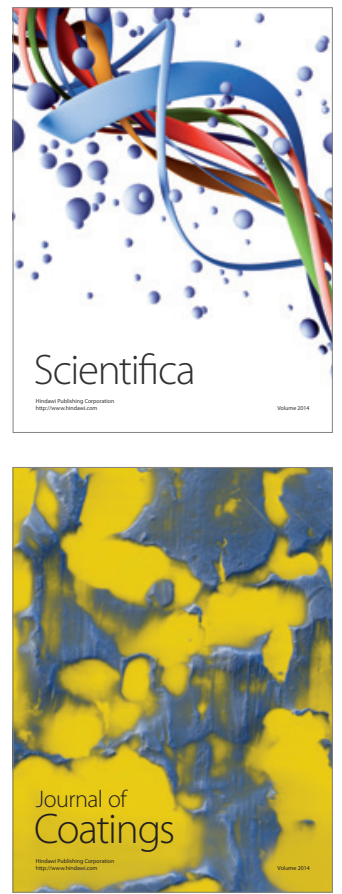
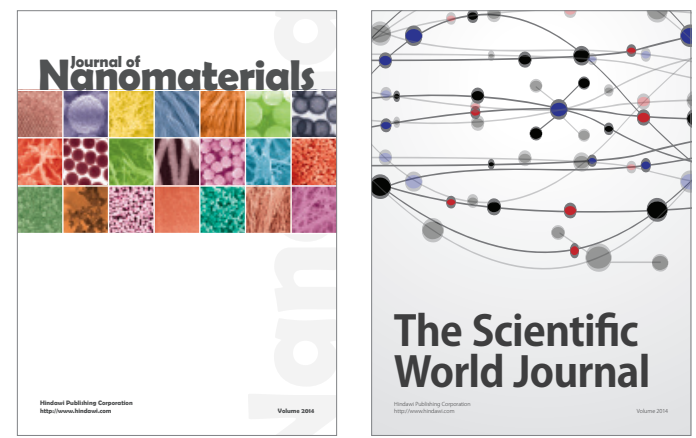

The Scientific World Journal
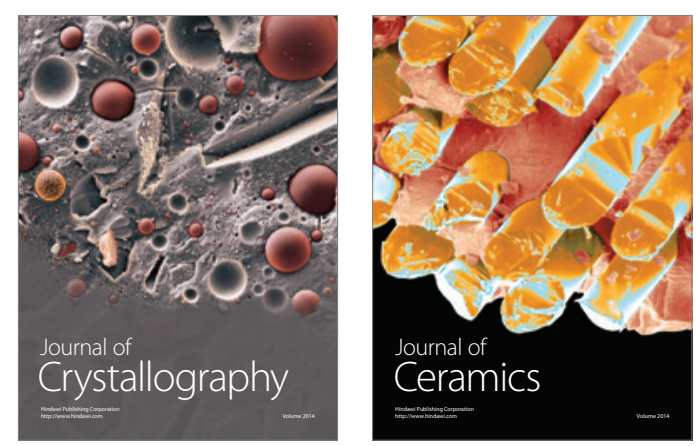
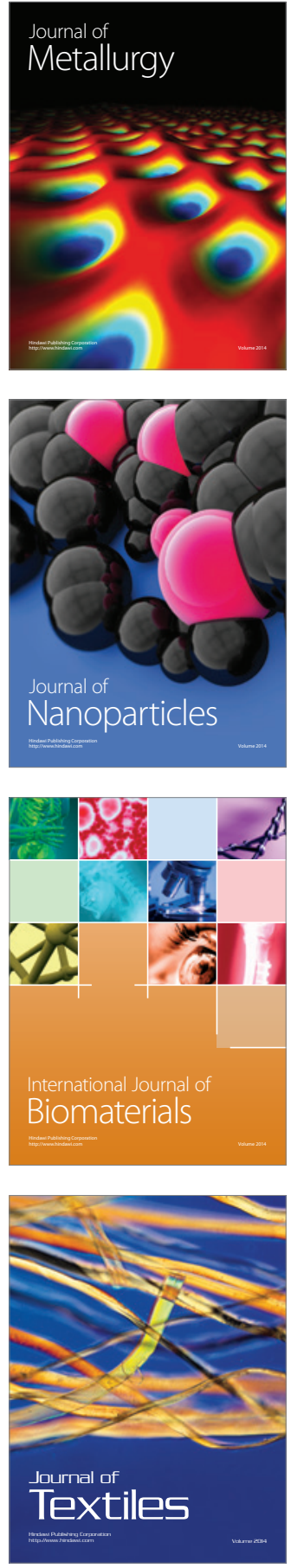